\title{
DESEMPENHO DA GESTÃO DE PORTFÓLIO DE PRODUTOS: PROPOSTA DE MODELO TEÓRICO CONCEITUAL APOIADO POR INTEGRAÇÃO INTERFUNCIONAL E SOCIALIZAÇÃO
}

Laura Maria Rafael (lauramaria@gmail.com) - Universidade Federal de São Carlos

Sergio Luis da Silva (sergiol.ufscar@gmail.com) - Universidade Federal de São Carlos

\section{RESUMO}

Um dos principais desafios enfrentados pela gestão de portfólio de produtos é a gestão das informações para a tomada de decisão de projetos. Alguns autores relatam que para facilitar a comunicação e a troca de informações nas relações organizacionais é necessário desenvolver a socialização, oriunda da gestão do conhecimento e facilitada pela integração interfuncional, a qual fortalece tais relações, desempenhando, portanto, importante papel na gestão de portfólio de produtos. Baseado em uma revisão bibliográfica sistemática, o presente trabalho apresenta um modelo teórico conceitual que relaciona as teorias de gestão de portfólio de produtos, integração interfuncional e socialização, tendo como característica a possível mediação parcial desta última entre a relação das duas primeiras teorias citadas. O modelo construído é uma contribuição para o estado da arte das teorias e para os segmentos empresariais porque diz respeito ao quanto as relações organizacionais, com ênfase em informações, podem influenciar de maneira direta aspectos relacionados à sobrevivência organizacional, como o processo de desenvolvimento de produtos e a correta inserção desses produtos em mercado.

Palavras chave: pré desenvolvimento de produtos; gestão de portfólio de produtos; integração interfuncional; gestão do conhecimento, socialização 


\section{INTRODUÇÃO}

O pré desenvolvimento é a base fundamental para o desenvolvimento bem sucedido de novos produtos, tendo como um dos pilares a gestão de portfólio de produtos (GPP) (KOEN et al., 2001), a qual engloba processos de tomada de decisão, incluindo revisões periódicas do portfólio com alocação de recursos estratégicos (COOPER; EDGETT; KLEINSCHMIDT, 1999; VOSS, 2012). No entanto, de acordo com Cooper, Edgett e Kleinschmidt (2000), um dos desafios enfrentados na GPP é obter informações para essa gestão.

Com relação a esse desafio, segundo Perks (2007), a concentração de poder em um ou poucos departamentos ou pessoas pode tornar a GPP mais difícil e, além disso, os dados podem estar indisponíveis, inconfiáveis ou incompletos, principalmente em ambientes considerados como tendo incertezas, caso do pré desenvolvimento (COOPER; EDGETT; KLEINSCHMIDT, 1999), prevalecendo a necessidade de comunicação para transferência de informações para o bom desempenho da GPP (MARLOW et al., 2018).

A fim de facilitar a comunicação e troca de informações, a literatura pontua a socialização, oriunda da gestão do conhecimento (GC), fundamental para as relações organizacionais (AALTONOEN; TURKULAINEN, 2018). De acordo com Cousins et al. (2008), mecanismos de socialização fornecem benefícios de natureza sustentável, principalmente por promoverem uma comunicação considerada aberta e limpa na organização. Os autores destacam, ainda, que a socialização é facilitada pela estrutura de integração interfuncional (IFF), de forma que a qualidade da troca de informações pode ser um indicativo da força das relações existentes. E, embora a IFF seja um tópico amplamente estudado, ainda há campo para crescimento quando se trata do pré desenvolvimento (PERKS, 2007).

Logo, devido ao desafio acerca de necessidades de informações, as incertezas e fluidez dos conhecimentos no pré desenvolvimento, valorizando a agilidade do conhecimento tácito, o objetivo desse artigo é apresentar um modelo teórico conceitual que sugere que a IFF apresente efeitos positivos no desempenho da GPP, tendo a socialização como possível mediadora parcial de tal relação.

O próximo tópico apresenta a fundamentação teórica acerca dos temas citados.

\section{REVISÃO TEÓRICA}

São apresentados nesse item as teorias de GPP, socialização e IFF e as interfaces que se percebe entre elas, as quais embasaram o desenvolvimento do modelo teórico conceitual. 


\subsection{Gestão de portfólio de produtos}

Em síntese, GPP trata de escolhas estratégicas de projetos selecionados para a execução, a continuação, despriorização ou mesmo o cancelamento dos já presentes no âmbito da empresa. Seu gerenciamento garante alocação de recursos de forma equilibrada com o número de projetos selecionados e sua consistência às estratégias empresariais, à maximização de valor econômico, ao balanceamento da carteira e à diminuição de riscos (COOPER; EDGETT; KLEINSCHMIDT, 1999; VOSS, 2012; JUGEND et al., 2017).

Os desafios relacionados à GPP estão apresentados no quadro 1.

QUADRO 1 - Problemas na gestão de portfólio de produtos. Fonte: Cooper, Edgett e Kleinschimidt (2000)

Problema

\begin{tabular}{|c|c|}
\hline Problema & No que diz respeito o problema \\
\hline Equilíbrio de recursos & $\begin{array}{c}\text { A demanda de recursos na maioria das empresas excede a sua oferta. } \\
\text { Dessa forma, é dificil o balanceamento das necessidades de recursos dos } \\
\text { projetos. }\end{array}$ \\
\hline Priorização de projetos & $\begin{array}{l}\text { Muitos projetos parecem ter potencial a primeira vista e acabam } \\
\text { entrando na lista ativa do portfólio por passarem por obstáculos impostos } \\
\text { pela tomada de decisões. A dificuldade é discriminar quais dos projetos } \\
\text { serão incluídos, mantidos e retirados da lista. }\end{array}$ \\
\hline Ausência de informações & $\begin{array}{l}\text { O trabalho inicial de levantamento de dados é, muitas vezes, abaixo do } \\
\text { necessário em projetos. Com isso, a administração é obrigada a tomar } \\
\text { decisões de investimentos significativas utilizando dados não confiáveis, } \\
\text { tornando tais decisões questionáveis. }\end{array}$ \\
\hline $\begin{array}{l}\text { Projetos pouco significativos } \\
\text { estrategicamente }\end{array}$ & $\begin{array}{l}\text { Há ausência de projetos com grande geração de receita, projetos que } \\
\text { beneficiam o avanço técnico e tecnológico e projetos que estão alinhados } \\
\text { com os setores financeiro e de mercado. }\end{array}$ \\
\hline
\end{tabular}

Considerando o foco desse trabalho como sendo a ausência de informações, percebe-se que as organizações vem promovendo a IFF como forma também de mitigar essas ausências, aumentando o número de ideias potenciais, flexibilidade da força de trabalho e o desempenho funcional de novos produtos, principalmente em ambientes com alta incerteza (CUJIPERS; GUENTER; HUSSINGER, 2011). A capacidade de uma empresa reduzir e lidar com incertezas está relacionada com a flexibilização de sua estrutura, como as relações interfuncionais, a qual expande a capacidade da troca e utilização das informações e conhecimento, notadamente entre pessoas, na forma tácita. Para tanto, o próximo tópico irá abordar conceitos a respeito da teoria de IFF. 


\subsection{Integração interfuncional}

A IFF, segundo o autor clássico Kahn (1996), é caracterizada como o conjunto de duas abordagens, a interação e colaboração.

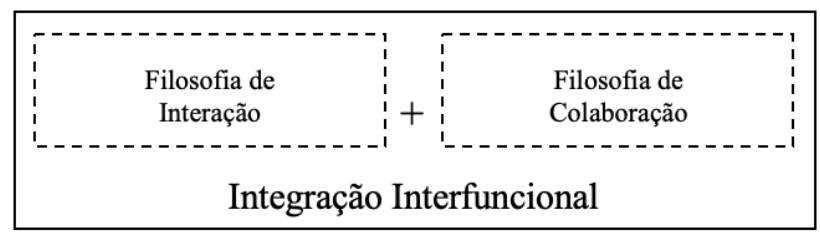

FIGURA 1 - Abordagens da IFF Fonte: Própria autora

A interação foca na comunicação convencional e atividades entre os indivíduos, sendo considerada como um fluxo formal de informações, como regularidade em reuniões e cuidados com documentação (KAHN, 1996).

Já a colaboração, representa uma relação de natureza afetiva, a qual minimiza atitudes e comportamentos negativos (GOMES et al., 2003). Kahn (1996) ainda destaca que, as atividades de colaboração são intangíveis, difíceis de sustentar sem que haja esforços coletivos e representa alto nível de relacionamento.

Dessa forma, o ponto central da IFF em processos que envolvam o desenvolvimento de produtos é que ela oferece ampla capacidade em obter, processar e interpretar informações de mercado, técnicas e financeiras (GOMES et al., 2003; GRIFFIN; HAUSER, 1996), o que traz maior eficácia com relação ao entendimento das necessidades dos clientes, pois permite compreensão por todos os envolvidos, bem como as possibilidades tecnológicas e capacidades empresariais (BUSS, 2002).

Logo, a fim de desenvolver ideias de produtos e avaliar suas tecnologias, características de produção, e viabilidade econômica, é necessário que a organização e seus indivíduos estejam aptos a superar diferenças, conflitos internos e barreiras impostas por alguns processos, implementando um ambiente colaborativo, mesmo tendo funções distintas (GOMES et al., 2003; GRIFFIN; HAUSER, 1996). A partir dessas características, é observada como a IFF corrobora com as necessidades da GPP.

Ademais, de acordo com Perks (2007), a natureza da IFF pode influenciar o sucesso do portfólio, assim observa-se a possível influência positiva da IFF no desempenho da GPP.

A literatura nos informa (NIJSSEN; VAN DER BORGH, 2017; XU et al., 2017) também que ao alcançar uma IFF mais consistente e madura, ou seja, mudança de perspectiva 
organizacional, automaticamente ocorre a socialização. Dessa forma, observa-se a possível influência positiva da IFF na socialização.

\subsection{Socialização}

A socialização é um processo de compartilhamento de experiências, onde um indivíduo pode adquirir conhecimento de outros, podendo ocorrer com observação, imitação ou prática. Tal processo considera a passagem do conhecimento tácito para tácito, informalmente, se referindo principalmente as percepções e aprendizados obtidos pelo sujeito a partir de algo novo ou pouco conhecido (TAKEUCHI; NONAKA, 2008).

A socialização é importante para a organização porque ajuda a garantir a continuidade de objetivos comuns, a similaridade de valores, as responsabilidades de cada membro e a interação entre eles (SMALE et al.; 2015), estando ligada aos aspectos da IFF.

Normalmente, na passagem de conhecimento tácito para tácito é necessário um ambiente propício para o desenvolvimento da comunicação entre indivíduos e departamentos funcionais de uma empresa. Silva (2002) afirma que os departamentos devem construir linguagens comuns, principalmente em processos que atuam conjuntamente, caso da GPP.

Alguns outros autores reforçam que o conhecimento tácito individual em situações de desenvolvimento produtos não é útil até que ocorra a facilitação para a sua transformação em coletivo e, que a integração entre os departamentos facilita a socialização (HIRUNYAWIPADA; BEYERLEIN; BLANKSON, 2010).

Dessa forma, como a socialização promove a comunicação aberta e limpa, bem como a troca de informações, fundamental para as relações organizacionais (AALTONOEN; TURKULAINEN, 2018; COUSINS, 2008), observa-se a possível mediação parcial da socialização na relação entre IFF e desempenho da GPP.

A seguir, será apresentada a revisão bibliográfica sistemática (RBS) realizada a qual fornece bases para o modelo teórico conceitual, o qual está esquematizado no quarto tópico do texto.

\section{METODOLOGIA}

Para construir a RBS, foi necessário definir as fontes primárias a partir de autores e periódicos clássicos do assunto pesquisado, que permitiu construir as strings de busca. Para as buscas, foi utilizada a base de dados mais Web of Science (WoS). Foram adicionadas às strings alguns 
termos sobre desenvolvimento de produtos, estratégia organizacional e competitividade a fim de ampliar a área estudada.

Com relação aos critérios de inclusão, sobre o tipo de documento, foram incluídos os artigos (de revistas, congressos, etc), livros e capítulos de livros. Sobre as restrições, foram retiradas da busca áreas de conhecimento que não ofereciam relação com os temas abordados, como medicina, por exemplo.

Após os filtros citados, em uma primeira rodada, foram obtidos 107 artigos. Em seguida, esses artigos foram passados por outros filtros, os quais estão dispostos na figura 2.

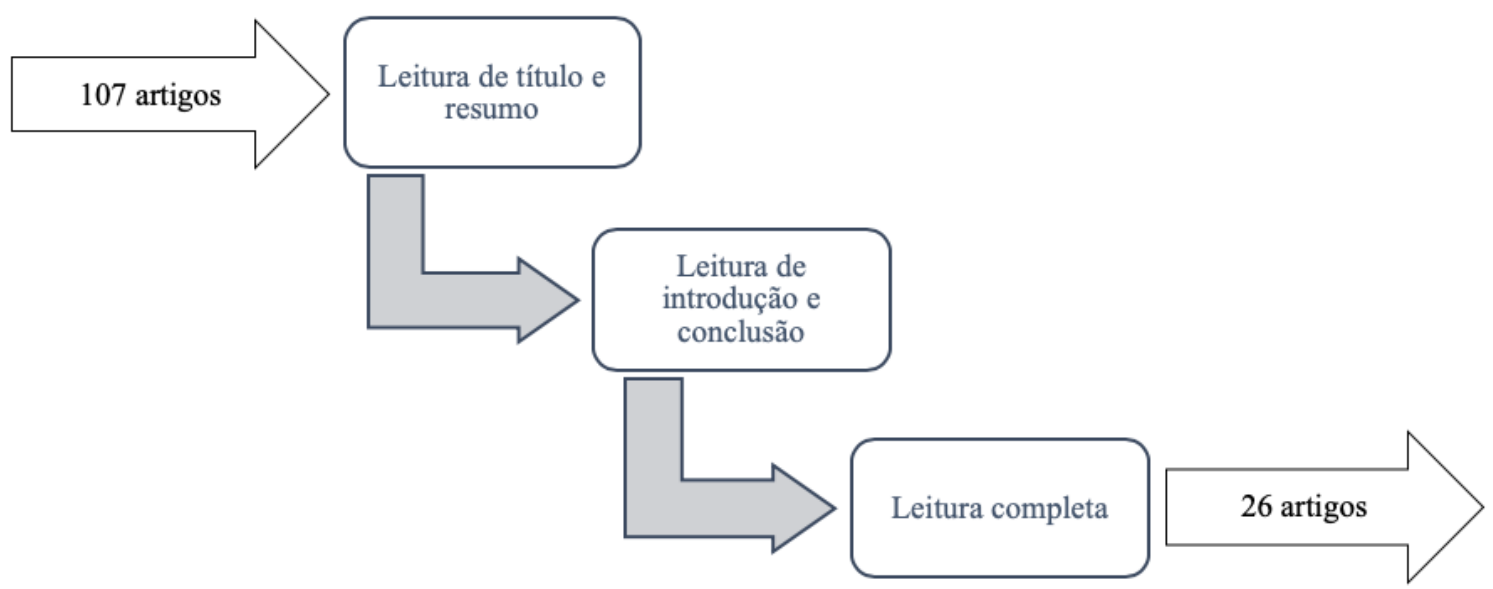

FIGURA 2 - Sequência de filtros da RBS. Fonte: Própria autora

Chegou-se a 26 artigos para a saída da RBS, sendo dois deles adicionados ao final do último filtro devido às suas citações serem constantes na maioria das leituras completas.

O próximo tópico discute com mais detalhes o modelo teórico conceitual e suas dimensões.

\section{RESULTADOS E DISCUSSÃO}

A partir da revisão teórica e da RBS, é apresentada a figura 3 com o modelo teórico conceitual acerca dos temas tratados no texto. 


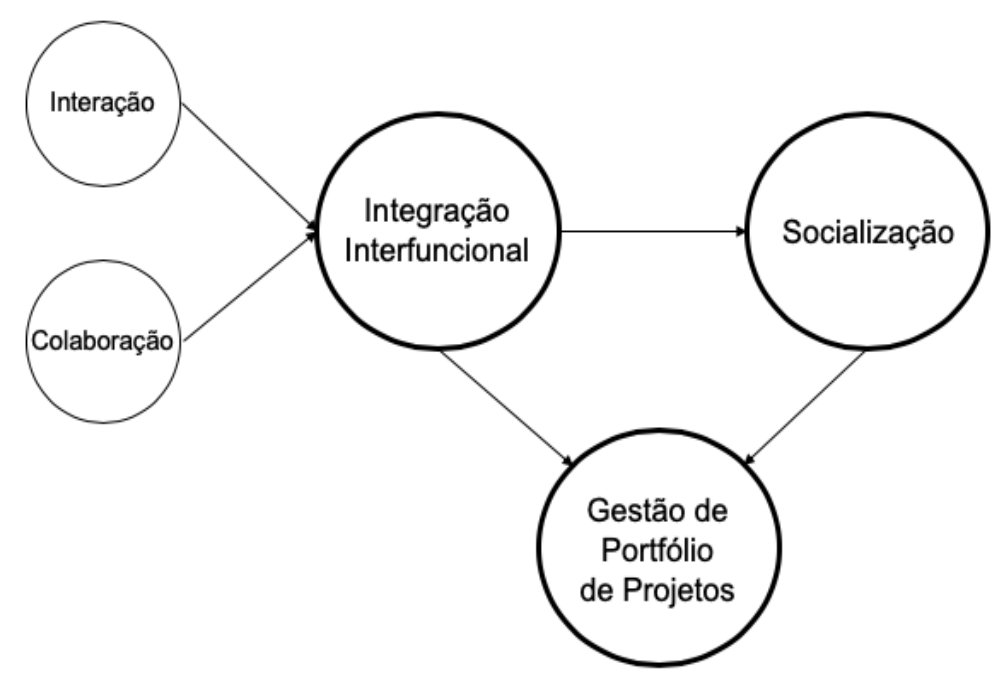

FIGURA 3 - Modelo teórico conceitual proposto. Fonte: Própria autora

Primeiro serão explicadas as práticas associadas à IFF (interação e colaboração) mais vistas na RBS e, em seguida, discussões que corroboram com as observações do modelo que foram expostas na revisão teórica. Destaca-se que o referencial teórico do item 2 é mais conceitual acerca das teorias que compõem o modelo. Já o presente item é o aprofundamento dessas teorias, de forma que foi construído com base na RBS.

\subsection{Integração interfuncional: Interação}

Uma das práticas de interação é o movimento de pessoal conhecido como job rotation, de forma que tal processo diminui a incerteza técnica, aumenta contatos e a comunicação, diminuindo as dificuldades de lidar com as diferenças culturais e formação entre os grupos (GRIFFIN; HAUSER, 1996; SONG; KAWAKAMI; STRINGFELLOW, 2010; FAIN; SCHOORMANS; DUHOVNIK, 2011; THOMÉ; SOUZA, 2016).

Outra prática, a proximidade física entre os departamentos, encoraja interações, trocas, comunicação e atração pessoal entre seus membros, maximizando a coesão entre eles e, consequentemente, a socialização. A separação pode fazer com que ocorram atrasos na tomada de decisão, podendo ser maléfica em contextos com mudanças como é a GPP (LEENDERS; WIERENGA, 2002).

Como a interação é caracterizada por comunicação formal, regras e procedimentos fazem parte de uma das práticas, elas se referem ao grau pelo qual atividades ou tarefas são controladas e, dessa forma, elas são integradas, atravessando barreiras entre os departamentos, o que mantém uma estrutura organizada com informações disponíveis. Mecanismos formais envolvendo 
passagem de fases e reuniões também são conhecidos por promoverem a socialização (SONG; KAWAKAMI; STRINGFELLOW, 2010; FAIN; SCHOORMANS; DUHOVNIK, 2011).

\subsection{Integração interfuncional: Colaboração}

Uma das práticas de colaboração é estabelecer objetivos em comum para todos os departamentos, minimizando conflitos gerados quando eles possuem interesses divergentes e não estejam alinhados, os quais podem colocar em cheque a GPP (GRIFFIN; HAUSER, 1996; SONG; KAWAKAMI; STRINGFELLOW, 2010). Xu, Qualls e Zang (2017) relacionam a socialização com o alinhamento de objetivos e valores entre os membros e ou departamentos da organização, apresentando relacionamento com a IFF. De acordo com Ghobadi e D'Ambra (2012), indivíduos são mais propensos a compartilhar seus conhecimentos e informações em contextos cooperativos, onde eles percebem o uso coletivo desse conhecimento compartilhado como busca de interesses comuns, de forma que esses autores relatam que há uma associação positiva entre essa cooperação na IFF e o compartilhamento de conhecimento.

Os sistemas sociais informais são uma prática que garante que as organizações criem mecanismos sociais como eventos e viagens para que facilite a transferência de informação entre seus colaboradores. Mantendo essas redes informais, pessoas podem compartilhar experiências ou expertise para solucionar problemas específicos, mantendo uns aos outros em contato durante o progresso da solução do problema (GRIFFIN; HAUSER, 1996; LEENDERS; WIERENGA, 2002).

A prática de estabelecer a confiança entre os membros dos diferentes departamentos também leva a potenciais resultados positivos na IFF, funcionando como uma forte força de coesão e altos níveis de colaboração. Por meio da confiança, os membros veem uns aos outros como competentes, responsáveis, pessoas abertas a novas ideias e propensos a trabalharem com propósitos semelhantes. Com altos níveis de confiança, os membros pedem por assistência, estimulando ideias criativas de colegas, estabelecendo ambiente de inclusão (JASSAWALLA; SASHITTAL, 1998). Aaltonoen e Turkulainen (2018) confirmam a necessidade da confiança para as relações organizacionais, acentuando a ideia de que coopera com a socialização. A partir desses recortes, a socialização e a IFF, mais uma vez, podem ser consideradas relacionadas. Kulangara, Jackson e Prater (2016) avaliaram o impacto da confiança entre compradores e fornecedores na socialização e no compartilhamento de informações em suas capacidades de inovação. $\mathrm{O}$ estudo mostrou que a socialização sem confiança não impacta a capacidade de 
inovação. Dessa forma, a promoção da confiança por meio da IFF está ligada com a melhoria da socialização e, consequentemente à troca de informações, a qual é necessária para o desempenho da GPP.

Atualmente, por exemplo, o envolvimento do cliente na GPP é gerado pelo departamento de marketing, sendo o representante desse cliente para os demais departamentos. Assim, a nível de portfólio, a integração entre departamento de marketing com outros torna-se fundamental (VOSS, 2012). Portanto, mais uma vez, a IFF aparece como papel importante para o alcance do bom desempenho da GPP.

\subsection{Socialização}

Nonaka, Toyama e Konno (2000) e Xu et al. (2017) apresentam indicativos empresariais que podem potencializar a socialização, como a ênfase na reunião de informação de diversas fontes e experiências e o compartilhamento de experiências oriundas de ambientes internos e externos à companhia, bem como a criação de novas ideias baseadas nesses ambientes. Além disso, treinamentos e workshops para melhorar as relações entre os colaboradores da companhia e a promoção de eventos sociais. Tais aspectos estão ligados as redes informais da colaboração e ao auxílio da movimentação de informações na empresa e, consequentemente à IFF e à GPP. Evidências na literatura mostram também que aspectos da socialização não ocorrem caso não exista o estabelecimento, entre os membros de uma organização, de objetivos comuns de longo prazo e recursos (XU; QUALLS; ZANG, 2017). Essas últimas características também relacionam a socialização com a IFF, na GPP.

Ademais, o conhecimento tácito pode ser disseminado com as chamadas redes informais, as quais são redes sociais de comunidades de práticas, nas quais há o compartilhamento de ideias, normas, etc, (ALMEIDA, 2016), sendo assim, a GPP tende a tornar-se mais eficiente quando ocorrem essas redes, pois propicia o aumento das informações na organização pelo aumento de confiança entre seus colaboradores. Kulangara, Jackson e Prater (2016) informam por meio da literatura evidências relacionando a IFF por meio da confiança com a transferência de conhecimento tácito para tácito (socialização) em contextos de inovação, o qual está inserido na GPP. 


\subsection{Gestão de portfólio de produtos}

De acordo com Cooper, Edgett e Kleinschmidt (2000), Bitman e Sharif (2008), Kester et al. (2011), um dos objetivos principais para a GPP é o alinhamento à estratégia de negócio, ou seja, promover que ocorra objetivos em comum entre os departamentos que participam de sua gestão, sendo necessária a IFF para ajudar a compor esse objetivo.

Huang, Chang e Henderson (2008) descreveram como os times relacionados ao processo de desenvolvimento de produtos podem reduzir as barreiras de alinhamento de estratégias por meio dos quatro passos de criação do conhecimento: socialização, externalização, combinação e internalização. O que se observa é a relação entre IFF e socialização descrevendo uma rota para facilitar a boa escolha de decisões de projetos na GPP com os objetivos da organização por meio da melhoria da comunicação e alinhamento entre os membros dos departamentos.

Quando se trata da GPP, o qual já foi destacado no texto como parte de um contexto com alto nível de incerteza, a IFF, segundo Cujipers, Guenter, Hussinger (2011), com sua alta capacidade de processamento de informações, aumenta a probabilidade de atendimento das necessidades do cliente. Nota-se novamente características complementares entre a GPP e a IFF.

$\mathrm{Na} \mathrm{GPP,} \mathrm{costuma} \mathrm{haver} \mathrm{o} \mathrm{envolvimento} \mathrm{de} \mathrm{muitas} \mathrm{áreas} \mathrm{funcionais} \mathrm{e} \mathrm{o} \mathrm{envolvimento} \mathrm{de} \mathrm{pessoas}$ de diferentes cargos, de forma que existem desafios relacionados à dificuldade em definir responsabilidades, bem como o uso de poder entre esses colaboradores, impactando de forma direta no gerenciamento de atividades (OLIVEIRA; ROZENFELD, 2010). Tal argumento corrobora que a IFF, por apoiar de maneira positiva objetivos em comum entre os departamentos organizacionais, minimiza o desafio da ausência de informações para a escolha correta de projetos.

\section{CONCLUSÃO}

A contribuição desse trabalho é um modelo teórico conceitual que diz respeito ao quanto as relações organizacionais podem influenciar de maneira direta aspectos de sobrevivência às companhias. Além disso, cobre uma lacuna na literatura, pois a IFF e a socialização, apesar de serem temas amplamente abordados, não há muitos estudos ou um bom desenvolvimento dessas teorias com o pré desenvolvimento, mais especificamente com a GPP. Em suma, foram encontradas evidências na literatura que mostram que o desempenho da GPP pode ser influenciado de maneira positiva pela IFF e, possivelmente mediada por um dos mecanismos 
da GC, a socialização, já que a mesma fortalece as relações entre os colaboradores e diferentes departamentos.

A partir da RBS, é importante destacar que, com relação às práticas de interação e de colaboração, existe a necessidade de equilíbrio entre meios de comunicação formais e informais para criação de novas ideias, bem como para a propensão à mudanças e, consequentemente à melhoria do fluxo de informações, a qual foi destacada no texto como sendo um dos desafios para a carteira de projetos.

Uma possível aplicação para pesquisas futuras sugere que tais temas, bem como o modelo teórico conceitual desenvolvido, poderiam ser aplicados, com o propósito de fornecer subsídios para a melhora da GPP e das relações organizacionais, em empresas com potencial inovador, as quais possuem grande volume de projetos em andamento e produtos sendo lançados em mercado, como é o caso dos setores alimentício, de telecomunicações ou de bens de capitais, por exemplo.

\section{AGRADECIMENTOS}

À Coordenação de Aperfeiçoamento de Pessoal de Nível Superior (CAPES) pelo auxílio financeiro para a realização desse trabalho.

\section{REFERÊNCIAS}

AALTONEN, K.; TURKULAINEN, V. Creating relational capital through socialization in project alliances. International Journal of Operations \& Production Management, v.38, n. 6, p. 1387-1421, 2018.

ALMEIDA, L. F. M. Um modelo para apoiar a gestão do conhecimento no gerenciamento ágil de projetos de software. [s.1.] Universidade Federal de São Carlos, 2016.

BUSS, C. O. Cooperação interfuncional no desenvolvimento de novos produtos: a interface marketingengenharia. [s.1.] Universidade Federal do Rio Grande do Sul, 2002.

COOPER, R. G.; EDGETT, S. J.; KLEINSCHMIDT, E. J. New product portfolio management: practices and performance. Journal of Product Innovation Management, v. 16, n. 4, p. 333-351, 1999.

COOPER, R. G.; EDGETT, S. J.; KLEINSCHMIDT, E. J. New Problems, New Solutions: Making Portfolio Management More Effective. Industrial Research Institute, Inc, v. 43, n. 2, p. 1-23, 2000.

COUSINS, P. D.; LAWSON, B.; SQUIRE, B. Performance measurement in strategic buyer-supplier relationships: the mediating role of socialization mechanismis. International Journal of Operations \& Production Management, v.28, n. 3, p. 238-258, 2008.

CUJIPERS, M.; GUENTER, H.; HURRINGER, K. Costs and benefits of inter-departmental innovation collaboration. Research Policy, v.40, n. , p. 565-575, 2011.

FAIN, N.; SCHOORMANS, J.; DUHOVNIK, J. The effect of R\&D-marketing integration on NPD success - the case of SMEs in the growing economy of Slovenia. International Journal of Technology Management. v. 56, n. 1, p. 92-107, 2011.

GHOBADI, S.; D’AMBRA, J. D. Knowledge sharing in cross-functional teams: a coopetitive model. Journal of 
Knowledge Management, v. 16, n. 2, p. 285-301, 2012.

GOMES, J. F. S. et al. Is more always better? An exploration of the differential effects of functional integration on performance in new product development. Technovation, v. 23, n., p. 185-191, 2003.

GRIFFIN, A.; HAUSER, J. R. Integrating R\&D and Marketing: A review and analysis of the literature. Journal of Product Innovation Management, v. 13, n. , p. 191-215, 1996.

HIRUNYAWIPADA, T.; BEYERLEIN, M.; BLANKSON, C. Cross-functional integration as a knowledge transformation mechanism : Implications for new product development. Industrial Marketing Management, v. 39 , p. 650-660, 2010.

HUANG, C. M.; CHANG, H. C.; HENDERSON, S. Knowledge transfer barriers between research and development and marketing groups within taiwanese small- and medium-sized enteprise high-technology new product development teams. Human Factors and Ergonomics in Manufacturing v. 18, n. 6, p. 621-657, 2008.

JASSAWALLA, A. R.; SASHITTAL, H. C. An Examination of Collaboration in High-Technology New Product Development Processes. Journal of Product Innovation Management, v. 15, n. , p. 237-254, 1998.

JUGEND et al. Green product development and product portfolio management: empirical evidence from an emerging economy. Business Strategy and the Environment. v. 26, n. , p. 1181-1195, 2017.

KAHN, K. B. Interdepartmental Integration: A definition with implications for product development performance. Journal of Product Innovation Management, v. 13, n. , p. 137-151, 1996.

KESTER, L. et al. Exploring portfolio decision-making processes. Journal of Product Innovation Management, v. 28, n. 5, p. 641-661, 2011.

KOEN, P. et al. Providing clarity and a common language to the "fuzzy front end". Research Technology Management, v. 44, n. 2, p. 46-55, 2001.

KULANGARA, N. P.; JACKSON, S. V.; PRATER, E. Examining the impact of socialization and information sharing and the mediating effect of trust on innovation capability. International Journal of Operations \& Production Management, v. 36, n. 11, p. , 2016.

MARLOW, S. L. et al. Does team communication represent a one-size-fits-all approach?: a meta-analysis of team communication and performance. Organizational Behavior and Human Decisions Process. v. , n. , p. , 2018.

NIJSSEN, E. J; VAN DER BORGH, M. Beyond the water cooler: using socialization to understanding use and impact of networking services on collaboration in a business incubator. Research and Development Management, v. , n. , p. , 2017.

NONAKA, I; TOYAMA, R; KONNO, N. SECI, Ba and Leadership: A unified model of dynamic knowledge creation. Long Range Plannig, v.33, n.1, p.5-34, 2000.

OLIVEIRA, M. G.; ROZENFELD, H. Integrating technology roadmapping and portfolio management at the frontend of new product development. Technological Forecasting \& Social Change, v. 77, n. , p. 1339-1354, 2010.

PERKS, H. Inter-functional integration and industrial new product portfolio decision making: Exploring and articulating the linkages. Creativity and Innovation Management, v. 16, n. 2, p. 152-164, 2007.

TAKEUCHI, H.; NONAKA, I. Gestão do Conhecimento. 1. ed. Porto Alegre: Bookman, 2008. 320 p.

THOMÉ, A. M. T.; SOUSA, R. Design-manufacturing integration and manufacturing complexity: a contingency investigation of job rotation and co-location. International Journal of Operations and \& Production Management. v. 36, n. 10, p. 1090-1114, 2016.

ROZENFELD, H. et al. Gestão de Desenvolvimento de Produtos: uma referência oara a melhoria do Processo. 1. ed. São Paulo: Editora Saraiva, 2006.

SILVA, S. L. Proposição de um modelo para caracterização das conversões do conhecimento no processo de desenvolvimento de produtos. [s.1.] Universidade de São Paulo, 2002.

SONG, M.; KAWAKAMI, T.; STRINGFELlOW, A. A Cross-National Comparative Study of Senior Management Policy, Marketing-Manufacturing Involvement, and Innovation Performance. Journal of Product Innovation Management, v. 27, n. , p. 179-200, 2010. 
VOSS, M. Impact of customer integration on project portfolio management and its success: developing a conceptual framework. International Journal of Project Management, v. 30, n. , p. 567-581, 2012.

$\mathrm{XU}$, L. et al. How socialization tacticts affect supplier-buyer co-development performance in exploratory and exploitative projects: the mediating effects of cooperation and collaboration. Journal of Business Research, v. , n. , p. , 2017. 